\title{
First-generation Corona Virus Disease 2019 (COVID-19) patients with a Wuhan-traveling history are prone to more severe diseases
}

\author{
Peiyan Zhang ${ }^{1}$, Jincheng Chen ${ }^{1}$, Guohui Xiao ${ }^{1}$, Peifen Chen ${ }^{2}$, Shuyang Liu ${ }^{1}$, Xiaoli Li $^{1}$, \\ Yinfeng $\mathrm{Li}^{1}$, Su Zhang ${ }^{1}$, Jing Bi $^{1}$, Shiyan Feng ${ }^{1}$, Fuxiang Wang ${ }^{1}$, Guoliang Zhang ${ }^{1}$, and \\ Yingxia Liu ${ }^{1}$
}

${ }^{1}$ Affiliation not available

${ }^{2}$ The Third People's Hospital of Shenzhen

June 11, 2020

\begin{abstract}
Wuhan has the highest number of deaths and mortality from COVID-19 in China to date, but the reason remains unknown. Here we compared patients with COVID-19 with a history of traveling in Wuhan with those who did not travel in Wuhan in the early stage of pandemic with respect to a series of clinical characteristics and laboratory parameters. We observed that patients who had traveled in Wuhan had an increased rate of severe disease and longer viral duration along with fewer CD4+ and CD8+ T cells, although there was no significant difference in viral load between the two groups.
\end{abstract}

\section{Introduction}

The outbreak of COVID-19, caused by SARS-CoV-2, in Wuhan city in China since December of 2019 has led to more than 80,000 infection cases and 4600 death cases in China and 4 million infection cases and 280,000 death cases worldwide ${ }^{1,2}$. Several papers have retrospectively analyzed the clinical characteristics of patients including children, the deceased, those who have recovered, and individuals with hypertension ${ }^{3-7}$. However, there are not yet reports on the comparison in clinical characteristics between patients with a travel history in Wuhan and patients without such experience in the early stage of pandemic.

In this study, we analyzed patients who had a history of traveling in Wuhan and patients who did not travel during January and February 2020 in Shenzhen Third People's Hospital. According to their clinical characteristics and laboratory parameters, we observed that patients with travel history in Wuhan had some differences in viral duration and $\mathrm{T}$ cell level when comparing with their counterparts.

\section{Methods}

We performed a retrospective review of the medical records of patients with COVID-19 who were admitted to Shenzhen Third People's Hospital during January and February 2020, after that the disease had spread to many cities in China, resulting in an inability to determine whether patients got virus infection in Wuhan or other places. In this study we defined the patients who had a history of traveling in Wuhan as WH patients, and those who did not have this history as NWH patients. We analyzed these two groups of patients for clinical characteristics, including cardio-pulmonary function, hepatic function, hemogram, immunologic parameters, viral load and duration, and severity of illness. This study was approved by the Ethics Committee of Shenzhen Third People's Hospital.

SPSS 18.0 and GraphPad Prism 7 were used to analyze the data, and $P<0.05$ was considered to be statistically significant. Continuous variables were expressed as the median and interquartile range and categorical variables were expressed as numbers and percentages. Unpaired t tests were used to compare continuous 
variables and the chi-square or Fisher's exact test was utilized to compare the proportions of the categorical variables.

\section{Results}

\section{Demographics and baseline characteristics of WH patients and NWH patients}

Fifty-nine patients who had a history of traveling in Wuhan and 53 patients who did not travel during January and February in 2020 were enrolled. As shown in Supplemental Table 1, female patients seemed to be more predominant in NWH patients $(30 / 53,56.6 \%)$ than in $\mathrm{WH}$ patients $(22 / 59,37.3 \%)$. The mean and median age of WH patients were 42.69 and 42 , respectively, which were higher than that of NWH patients, but there was no significant difference. WH patients had a similar percentage of comorbidities with NWH patients. The incubation period of SARS-Cov-2 was similar in these two groups (9.44 d vs 9.08 d). WH patients seemed to have a higher percentage of shivering, cough, nasal obstruction, and sore throat than NWH patients. We also observed that the percentage of severe to critical cases in WH patients was significantly higher than that in NWH patients $(\mathrm{P}<0.05)$ (Figure $1 \mathrm{~A})$. In addition, two death cases occurred in WH patients.

\section{Laboratory parameters of WH and NWH patients}

We next compared laboratory data of these two groups of patients at hospital admission, midterm (7-15 days after their admission), and discharge. We observed that there were no obvious differences in pulmonary function parameters although the level of lactic acid was higher in WH patients at hospital admission and discharge (Supplemental Figure 1). Although there were no significant differences in the levels of total bilirubin, direct bilirubin, alanine aminotransferase (ALT) and glutamic oxalacetic transaminase (AST), the levels of blood urea nitrogen (BUN) and creatinine (Crea) were increased in WH patients at the hospital midterm and admission respectively (Supplemental Figure 2). From the measurement of blood coagulation, we observed a significantly longer time of activated partial thromboplastin time (APTT) in WH patients at hospital admission and midterm (Supplemental Figure 3). Procalcitonin (PCT) and C reactive protein (CRP) and interleukin-6 (IL-6) were not significantly different between the two groups (Supplemental Figure 4). The viral load of the two groups had no significant difference at hospital admission, and midterm (Figure 1B), but the viral duration of WH patients was longer than that of NWH patients $(\mathrm{P}<0.05)$ (Figure $1 \mathrm{C})$. In the hemogram record, WH patients had lower lymphocyte counts than NWH patients at hospital midterm and lower platelet counts at hospital admission and midterm (Supplemental Figure 5). We further found that WH patients had lower levels of $\mathrm{CD}^{+}, \mathrm{CD}^{+}$and $\mathrm{CD} 8^{+} \mathrm{T}$ cells in peripheral blood than $\mathrm{NWH}$ patients at all the three checks, hospital midterm and discharge, and hospital midterm respectively (Figure 2A-C).

\section{Discussion}

This present study showed that COVID-19 patients with a history of traveling in Wuhan had some clinical characteristics and laboratory parameters that were different from COVID-19 patients who did not travel in Wuhan. Lymphocytopenia, including the reduced $\mathrm{CD} 3^{+}, \mathrm{CD} 4^{+}$and $\mathrm{CD} 8^{+} \mathrm{T}$ cell counts on hospital midterm were observed in Wuhan patients and percentage of severe to critical cases in WH patients was significantly higher than that in NWH patients, implying decrease in lymphocyte was related to the severity of the disease, which is in agreement with the statement by Zhang et al ${ }^{8}$. The observation that patients with COVID-19 with a history of traveling in Wuhan more prone to severe diseases could be explained from two aspects, firstly, SARS-CoV-2 might evolve adaptations to optimize its fitness to novel hosts during human-to-human transmission, and viral adaptation may increase its transmissibility but attenuate its pathogenesis, similar pattern have also been observed in Ebola virus ${ }^{9}$. Since mutations are part of the convergent evolution, it would be interesting to investigate the dynamic intra-host genomic variations of SARS-CoV-2 during the transmission process of COVID-19 patients. Secondly, after the outbreak in Wuhan, the local CDC strengthened the screen of COVID-19 patients, especially those with a traveling history to Wuhan and close contacts with confirmed cases, early identification and treatment is an efficient tactics to avoid the development of severe diseases. 


\section{Acknowledgments}

The authors thank all the staff working in the Department of Infectious Diseases from Shenzhen Third People's Hospital for providing COVID-19 clinical data.

Funding This work was supported by the National Science and Technology Major Project for Control and Prevention of Major Infectious Diseases of China (No. 2017ZX10103004), the Guangdong Scientific and Technological Project (NO. 2020B1111340076), and the Shenzhen Bay Laboratory Open Project (No. SZBL202002271003).

\section{Conflicts of interest}

The authors declared no conflict of interest.

\section{References}

1. Zhu N, Zhang D, Wang W, et al. A novel coronavirus from patients with pneumonia in China, 2019. New England Journal of Medicine. 2020.

2. Wu Z, McGoogan JM. Characteristics of and important lessons from the coronavirus disease 2019 (COVID19) outbreak in China: summary of a report of 72314 cases from the Chinese Center for Disease Control and Prevention. Jama. 2020;323(13):1239-1242.

3. Liu W, Zhang Q, Chen J, et al. Detection of Covid-19 in children in early January 2020 in Wuhan, China. New England Journal of Medicine. 2020;382(14):1370-1371.

4. Chen T, Wu D, Chen H, et al. Clinical characteristics of 113 deceased patients with coronavirus disease 2019: retrospective study. Bmj.2020;368.

5. Lan L, Xu D, Ye G, et al. Positive RT-PCR test results in patients recovered from COVID-19. Jama. 2020;323(15):1502-1503.

6. Meng J, Xiao G, Zhang J, et al. Renin-angiotensin system inhibitors improve the clinical outcomes of COVID-19 patients with hypertension. Emerging microbes $\& 3$ infections. 2020;9(1):757-760.

7. Yanrong W, Yingxia L, Lei L, Xianfeng W, Nijuan L, Ling L. Clinical Outcomes in 55 Patients With Severe Acute Respiratory Syndrome Coronavirus 2 Who Were Asymptomatic at Hospital Admission in Shenzhen, China. The Journal of Infectious Diseases. 2020.

8. Zhang X, Tan Y, Ling Y, et al. Viral and host factors related to the clinical outcome of COVID-19. Nature. 2020:1-7.

9. Urbanowicz RA, McClure CP, Sakuntabhai A, et al. Human adaptation of Ebola virus during the West African outbreak. Cell.2016;167(4):1079-1087. e1075.

\section{Figure legends}

Figure 1 Disease severity, viral load, and viral duration in WH and NWH patients. (A) Disease severity distribution of the two groups during hospitalization. (B) Viral load at hospital admission and midterm in WH and NWH patients. (C) Viral duration in WH and NWH patients. The viral duration was determined as the time from patient's exposure to other confirmed patients until his/her viral nucleic acid test was negative. The data are expressed as the median and IQR. An unpaired t test was used, and $\mathrm{P}<0.05$ was considered significant.

Figure $2 \mathrm{~T}$ cell count in peripheral blood from $\mathbf{W H}$ and $\mathbf{N W H}$ patients. (A) $\mathrm{CD} 3^{+} \mathrm{T}$ cell count in peripheral blood $/ \mu \mathrm{L}$. (B) $\mathrm{CD} 4^{+} \mathrm{T}$ cell count in peripheral blood $/ \mu \mathrm{L}$. (C) $\mathrm{CD} 8^{+} \mathrm{T}$ cell count in peripheral blood $/ \mu \mathrm{L}$. The data are expressed as the median and IQR. An unpaired t test was used, and $\mathrm{P}<0.05$ was considered significant. 
Supplemental Figure 1 Pulmonary function in WH and NWH patients at different time points . (A) pH. (B) Partial pressure of oxygen (PO2), mmHg. (C) Partial pressure of carbon dioxide(PCO2), mmHg. (D) Fraction of inspiration O2(FIO2), \% . (E) Oxygenation index(OI), mmHg. The data are expressed as the median and IQR. An unpaired t test was used, and $\mathrm{P}<0.05$ was considered significant.

Supplemental Figure 2 Hepatic function in WH and NWH patients at different time points. (A) Total bilirubin(TBil), $\mu \mathrm{mol} / \mathrm{L}$. (B) Direct bilirubin(DBil), $\mu \mathrm{mol} / \mathrm{L}$. (C) Alanine aminotransferase(ALT), U/L. (D) Glutamic oxalacetic transaminase(AST), U/L. (E) Lactic dehydrogenase(LDH), U/L. (F) Blood urea nitrogen(BUN), mmol/L. $(\mathrm{G})$ Creatinine(Crea), $\mu \mathrm{mol} / \mathrm{L}$. The data are expressed as the median and IQR. An unpaired t test was used, and $\mathrm{P}<0.05$ was considered significant.

Supplemental Figure 3 Blood coagulation function in WH and NWH patients at different time points. (A) plasma prothrombin time(PT), s. (B) activated partial thrombin(APTT), s. (C) Fibrinogen(FIB), g/L. (D) D-Dimer, $\mu \mathrm{g} / \mathrm{mL}$. The data are expressed as the median and IQR. An unpaired t test was used, and $\mathrm{P}<0.05$ was considered significant.

Supplemental Figure 4 Inflammatory indicators in WH and NWH patients at different time points. (A) Procalcitonin (PCT), ng/mL. (B) C reactive protein (CRP), mg/L. (C) Interleukin-6(IL-6), $\mathrm{pg} / \mathrm{mL}$. The data are expressed as the median and IQR. An unpaired t test was used, and $\mathrm{P}<0.05$ was considered significant.

Supplemental Figure 5 Hemogram in $\mathrm{WH}$ and $\mathrm{NWH}$ patients at different time points . (A) White blood cell count(WBC), ${ }^{*} 10^{9} / \mathrm{L}$. (B) Lymphocyte count(Lymph), ${ }^{*} 10^{9} / \mathrm{L}$. (C) Neutrophil count(Neut), ${ }^{*} 10^{9} / \mathrm{L}$. (D) Eosinophils count, ${ }^{*} 10^{9} / \mathrm{L}$. (E) haemoglobin(Hgb), g/L. (F) platelet count(PLT), $* 10^{9} / \mathrm{L}$. (F) Albumin(ALB), g/L. The data are expressed as the median and IQR. An unpaired t test was used, and $\mathrm{P}<0.05$ was considered significant.

A

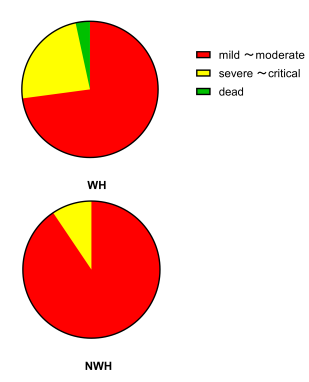

A

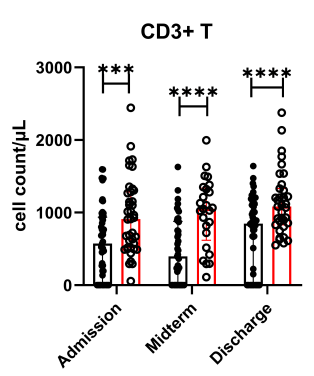

B

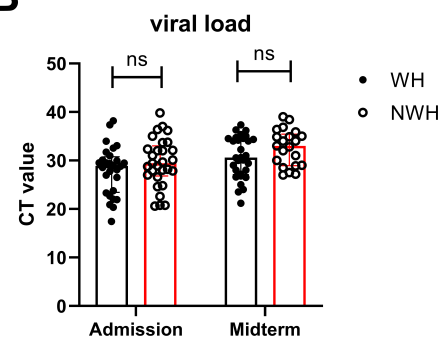

C

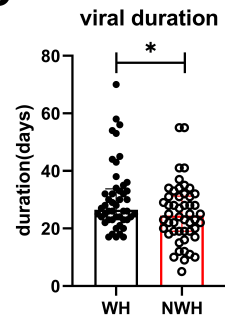

B

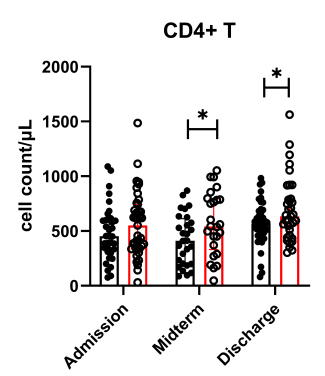

C

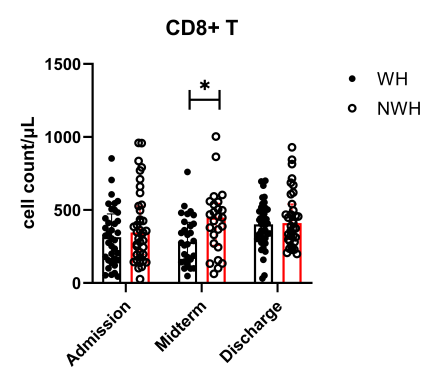

\section{Hosted file}

supplemental-table-1-20200603.docx available at https://authorea.com/users/332377/articles/ 458833-first-generation-corona-virus-disease-2019-covid-19-patients-with-a-wuhan- 
traveling-history-are-prone-to-more-severe-diseases

\section{Hosted file}

supplemental-figure.docx available at https://authorea.com/users/332377/articles/458833first-generation-corona-virus-disease-2019-covid-19-patients-with-a-wuhan-travelinghistory-are-prone-to-more-severe-diseases 